\title{
Review: artesunate added to standard drug treatment reduces treatment failure in malaria
}

International Artemisinin Study Group. Artesunate combinations for treatment of malaria: meta-analysis. Lancet 2004;363:9-17.

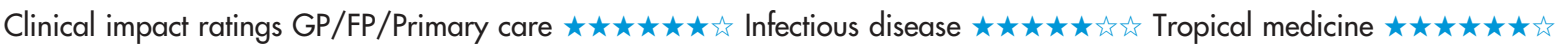

In patients with acute, uncomplicated Plasmodium falciparum malaria, does the addition of an artemisinin derivative (artesunate) to existing drug treatment reduce treatment failure and transmission potential?

\section{METHODS}

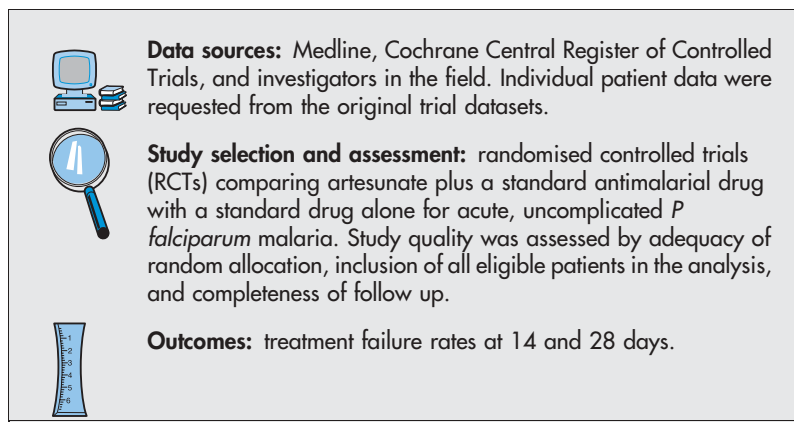

\section{MAIN RESULTS}

16 trials met the selection criteria $(\mathrm{n}=5948)$. The background drugs used were chloroquine ( 3 RCTs done in Burkina Faso, Ivory Coast, and Sao Tome and Principe), amodiaquine (3 RCTs done in Gabon, Kenya, and Senegal), sulphadoxine-pyrimethamine (7 RCTs done in Gambia, Kenya, Malawi, Peru, and Uganda), and mefloquine (3 RCTs done in Thailand). 12 RCTs were placebo controlled. All but 1 RCT assessed 3 days of artesunate. 3 days of artesunate added to background treatment lowered treatment failure rates more than background treatment alone at 14 and 28 days (table). Gametocytaemia was also reduced with 3 days of artesunate (11 RCTs) (odds ratio at $28 \mathrm{~d} 0.04,95 \%$ CI 0.02 to 0.08 ).

For correspondence: Professor P Garner, Liverpool School of Tropica Medicine, Liverpool, UK. pgarner@liv.ac.uk

Sources of funding: World Health Organization and Department for International Development UK.

\section{CONCLUSION}

In patients with acute, uncomplicated Plasmodium falciparum malaria, the addition of artesunate to existing drug treatment reduces treatment failure.

\section{Commentary}

7 he studies analysed in the review by the International Artemisinin Study Group focus on the reduction of the development of drug resistant malaria in endemic areas. In addition to improving clinical outcomes in malaria endemic countries, the data are also pertinent to practitioners in non-endemic areas because travel to and immigration from these areas are common and imported cases of malaria are seen in practice.

The methods used in this analysis are sound, and the conclusions drawn are reliable. Artesunate, a derivative of a natural product, sweet wormwood, was first developed in China as an antimalarial. The World Health Organization recommends that an artemisinin derivative is included in antimalarial treatment regimens both to improve the clinical response and cure rate and to slow the development of malaria resistance. ${ }^{1}$ Because artesunate works rapidly to kill most malarial parasites, it leads to a more rapid resolution of fever and can help prevent malaria complications. ${ }^{2} 3$ The longer half life companion drug kills the malaria parasites that remain.

This product is not currently licensed in the US and therefore may have limited availability. Future research is focused on developing fixed dose combinations including artesunate for treatment of malaria. Mary M Fanning, MD, PhD National Institute of Allergy and Infectious Diseases National Institutes of Health Bethesda, Maryland, USA

1 World Health Organization. Antimalarial Drug Combination Therapy Report of a WHO Technical Consultation. WHO unpublished report WHO/ CDS/RBM/2001.35. Geneva: WHO, 2001

2 Wattanakoon $Y$, Chittamas S, Pornkulprasit $V$, et al. Six-years monitoring the efficacy of the combination of artesunate and mefloquine for the treatment of uncomplicated falciparum malaria. Southeast Asian J Trop Med Public Health 2003;34:542-5.

3 Mohanty AK, Rath BK, Mohanty R, et al. Randomized control trial of quinine and artesunate in complicated malaria. Indian J Pediatr 2004;71:291-5.

4 Olliaro PL, Taylor WR. Developing artemisinin based drug combinations for the treatment of drug resistant falciparum malaria: A review. J Postgrad Med 2004;50:40-4.

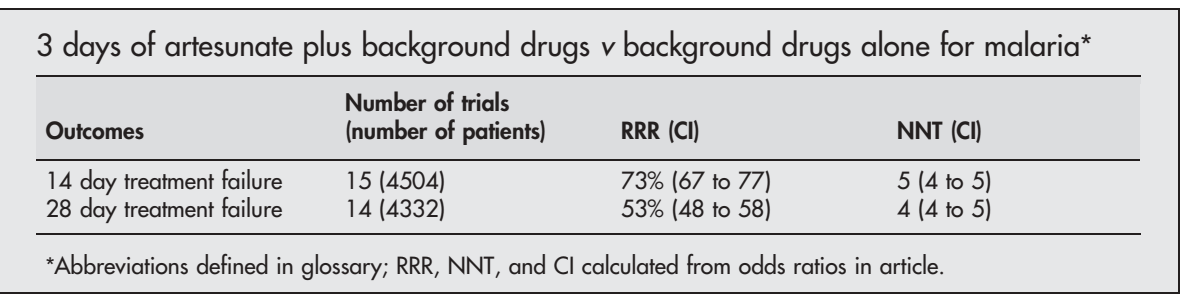

